\title{
p53 isoform $\Delta 113$ p53 promotes zebrafish heart regeneration by maintaining redox homeostasis
}

\author{
Shengfan Ye', Ting Zhao', Wei Zhang ${ }^{1}$, Zimu Tang ${ }^{1}$, Ce Gao², Zhipeng Ma', Jing-Wei Xiong ${ }^{3}$, Jinrong Peng ${ }^{2}$, \\ Wei-Qiang Tan $\mathbb{B}^{4}$ and Jun Chen ${ }^{1}$
}

\begin{abstract}
Neonatal mice and adult zebrafish can fully regenerate their hearts through proliferation of pre-existing cardiomyocytes. Previous studies have revealed that p53 signalling is activated during cardiac regeneration in neonatal mice and that hydrogen peroxide $\left(\mathrm{H}_{2} \mathrm{O}_{2}\right)$ generated near the wound site acts as a novel signal to promote zebrafish heart regeneration. We recently demonstrated that the expression of the p53 isoform $\triangle 133 p 53$ is highly induced upon stimulation by low-level reactive oxygen species (ROS) and that $\triangle 133 p 53$ coordinates with full-length p53 to promote cell survival by enhancing the expression of antioxidant genes. However, the function of p53 signalling in heart regeneration remains uncharacterised. Here, we found that the expression of $\triangle 113 p 53$ is activated in cardiomyocytes at the resection site in the zebrafish heart in a full-length p53- and ROS signalling-dependent manner. Cell lineage tracing showed that $\Delta 113 p 53$-positive cardiomyocytes undergo cell proliferation and contribute to myocardial regeneration. More importantly, heart regeneration is impaired in $\triangle 113 p 53^{M / M}$ mutant zebrafish. Depletion of $\Delta 113 p 53$ significantly decreases the proliferation frequency of cardiomyocytes but has little effect on the activation of gata4positive cells, their migration to the edge of the wound site, or apoptotic activity. Live imaging of intact hearts showed that induction of $\mathrm{H}_{2} \mathrm{O}_{2}$ at the resection site is significantly higher in $\triangle 113 p 53^{M / M}$ mutants than in wild-type zebrafish, which may be the result of reduced induction of antioxidant genes in $\triangle 113 p 53^{M / M}$ mutants. Our findings demonstrate that induction of $\triangle 113 p 53$ in cardiomyocytes at the resection site functions to promote heart regeneration by increasing the expression of antioxidant genes to maintain redox homeostasis.
\end{abstract}

\section{Introduction}

The adult mammalian heart has limited regenerative capability following cardiac damage, and this is the main reason that cardiac infarction is one of the leading causes of death worldwide ${ }^{1}$. In contrast, the hearts of adult zebrafish and neonatal mice exhibit full cardiac regeneration capacity following ventricular resection or cryoinjury through robust cardiomyocyte proliferation ${ }^{2-4}$.

\footnotetext{
Correspondence: Wei-Qiang Tan (tanweixxxx@zju.edu.cn) or

Jun Chen (chenjun2009@zju.edu.cn)

'MOE Key Laboratory of Biosystems Homeostasis \& Protection and Innovation Center for Cell Signaling Network, College of Life Sciences, Zhejiang University, 310058 Hangzhou, China

${ }^{2}$ College of Animal Sciences, Zhejiang University, 310058 Hangzhou, China

Full list of author information is available at the end of the article

These authors contributed equally: Shengfan Ye, Ting Zhao

Edited by I. Amelio
}

In zebrafish, cardiomyocytes from the subepicardial ventricular layer dedifferentiate into gata4-positive cardiomyocytes to proliferate and invade the area of injury, and this is the major process underlying heart regeneration ${ }^{5,6}$.

A number of signalling pathways, including the Notch, BMP, PDGF, RA, Nrg1 and Brg1 pathways, have been documented to regulate zebrafish cardiac regeneration ${ }^{7-15}$. Reactive oxygen species (ROS), specifically $\mathrm{H}_{2} \mathrm{O}_{2}$, produced in the epicardium and adjacent myocardium near the wound site have also been found to promote the proliferation of cardiomyocytes ${ }^{16}$. ROS, including superoxide anion $\left(\mathrm{O}_{2}{ }^{-}\right)$, hydroxyl radical $\left(\mathrm{OH}^{*}\right)$ and the nonradical species hydrogen peroxide $\left(\mathrm{H}_{2} \mathrm{O}_{2}\right)$, play a dual role in cell fate determination. At moderate levels, ROS can function as signals that promote cell growth and divi$\operatorname{sion}^{17-19}$. In contrast, when ROS are overproduced

\section{(c) The Author(s) 2020}

(c) (i) Open Access This article is licensed under a Creative Commons Attribution 4.0 International License, which permits use, sharing, adaptation, distribution and reproduction c. in any medium or format, as long as you give appropriate credit to the original author(s) and the source, provide a link to the Creative Commons license, and indicate if changes were made. The images or other third party material in this article are included in the article's Creative Commons license, unless indicated otherwise in a credit line to the material. If material is not included in the article's Creative Commons license and your intended use is not permitted by statutory regulation or exceeds the permitted use, you will need to obtain permission directly from the copyright holder. To view a copy of this license, visit http://creativecommons.org/licenses/by/4.0/. 
beyond a cell's capacity to maintain redox homeostasis, they can lead to oxidation of macromolecules such as proteins, membrane lipids and mitochondria and genomic $\mathrm{DNA}^{20,21}$. The harmful accumulation of ROS eventually results in abnormal cell death and senescence.

To maintain redox homeostasis, organisms have evolutionarily developed numerous antioxidant defence systems, including both enzymatic and non-enzymatic antioxidant mechanisms that can either scavenge ROS or prevent their formation ${ }^{22}$. In response to oxidative stress, the signalling pathway of the tumour repressor p53 plays important and complex roles ${ }^{23-26}$. Under physiological conditions and during low levels of oxidative stress, p53 functions to maintain oxidative homeostasis and promote cell survival through transcriptionally expressing antioxidant genes $^{27-32}$. However, p53 triggers apoptotic activity by upregulating the expression of pro-oxidative genes and apoptotic genes in response to high levels of oxidative stress $^{30,33-35}$. Zebrafish $\Delta 113 \mathrm{p} 53$ and its human counterpart $\Delta 133 \mathrm{p} 53, \mathrm{~N}$-terminal truncated isoforms of p53, are both transcribed by an alternative $p 53$ promoter in intron $4^{36,37}$. Full-length p53 can directly transactivate the transcription of these isoforms in response to both developmental and DNA damage stresses ${ }^{38-40}$. In turn, the induction of $\Delta 113 \mathrm{p} 53 / \Delta 133 \mathrm{p} 53$ inhibits p53dependent apoptosis by differentially modulating the expression of p53 target genes ${ }^{36,37,40} . \Delta 113$ p53/4133p53 can form a complex with p53 both in vitro and in vivo, and this interaction is essential for its anti-apoptotic activity $^{41}$. The basal expression of $\Delta 133 \mathrm{p} 53$ prevents normal human fibroblasts, T-lymphocytes and astrocytes from p53-mediated replicative senescence by repressing $m i R-34 a$ expression ${ }^{42,43}$. In response to $\gamma$-irradiation, $\Delta 113 p 53 / \Delta 133 p 53$ not only represses cell apoptosis but also coordinates with p73 to promote DNA DSB repair by upregulating the transcription of repair genes ${ }^{44,45}$. Interestingly, our recent study revealed that upon treatment with sub-toxic ROS stresses, $\Delta 133$ p53 does not antagonise the activity of p53 but coordinates with p53 to promote cell survival by promoting antioxidant gene expression $^{46}$.

A study in mice showed that p53 signalling is activated in cardiomyocytes during neonatal mouse heart regeneration ${ }^{47}$. However, the roles p53 signalling plays and whether its isoforms are activated in heart regeneration are unknown. In this report, we reveal that $\triangle 113 p 53$ is induced in cardiomyocytes at the resection site in the zebrafish heart and that this induction is dependent on full-length p53 and ROS signalling. Furthermore, $\Delta 113$ p53 promotes heart regeneration through upregulating the expression of antioxidant genes. Our results demonstrate that activation of the p53 signalling pathway is required for heart regeneration by maintaining redox homeostasis.

\section{Results}

The expression of $\Delta 113 p 53$ is induced in cardiomyocytes at the resection site in the zebrafish heart

To investigate whether the p53 signalling pathway is also activated during zebrafish heart regeneration as in neonatal mice, we surgically removed $\sim 15 \%$ of ventricular cardiomyocytes from $\operatorname{tg}(\triangle 113 p 53: G F P)$ transgenic zebrafish, in which the expression of GFP faithfully mimics the transcription of endogenous $\Delta 113 p 53^{40}$. Interestingly, we found that the GFP signal was co-localised with MHC (the myosin heavy chain of cardiomyocytes) at the resection site beginning 7 days post-amputation (dpa; Fig. 1c), reached a peak at $21 \mathrm{dpa}$ and decreased at $30 \mathrm{dpa}$ (Fig. 1d-f), whereas the green fluorescent signal was merely observed in the ventricles of both the sham hearts and the resected hearts at $4 \mathrm{dpa}$ (Fig. 1a, b, g).

To confirm the activation of the p53 signalling pathway, we performed an in situ hybridisation assay with a probe that detects both full-length $p 53$ and $\Delta 113 p 53$. Positive signals were observed in cells near the resection site in wild-type (WT) hearts at $14 \mathrm{dpa}$ (Fig. 1i) but not in the ventricles in sham hearts (Fig. 1h). Quantitative reverse transcription PCR (qRT-PCR) showed that the expression of $\Delta 113 p 53$ and $p 21$ (also a p53 target gene), but not fulllength $p 53$, was significantly increased in the resected hearts compared to the sham hearts at $7 \mathrm{dpa}$ (Fig. 1j).

As $4113 p 53$ is a p53 target gene, we asked whether the induction of the transgene was p53-dependent. For this purpose, the $\operatorname{tg}(\triangle 113 p 53: G F P)$ transgene was crossed into the $p 53^{M 214 K}$ mutant background, in which the transcriptional activity of mutant p53 is lost ${ }^{48}$. Unlike in the resected hearts of WT fish, GFP was not detectable in the resected hearts of $p 53^{M 214 K}$ mutant fish at $14 \mathrm{dpa}$ (Figs. S1 and $1 \mathrm{k}, \mathrm{l})$. Taken together, the results suggest that fulllength p53 was post-transcriptionally activated to upregulate the expression of its downstream genes, including $\Delta 113 p 53$, during heart regeneration.

\section{$\Delta 113 p 53$-positive cardiomyocytes undergo cell proliferation and contribute to heart regeneration}

To explore whether the induction of $\triangle 113 p 53$ is related to the proliferation of cardiomyocytes, we subjected $t g$ ( $\triangle 113 p 53: G F P)$ zebrafish to EdU (5-ethynyl-2'-deoxyuridine)-labelling from 5 to $7 \mathrm{dpa}$. At $7 \mathrm{dpa}, \sim 4.3 \%$ of cardiomyocytes (MF20-positive cells) in the wound area were labelled with Edu (Fig. 2b-d), whereas up to $10.2 \%$ of $\Delta 113 p 53$-positive cardiomyocytes were labelled with Edu (Fig. 2e). The Edu-labelled $\Delta 113 p 53$-positive cardiomyocytes accounted for $24.4 \%$ of total Edu-labelled cardiomyocytes (Fig. S2). The Edu-labelled cardiomyocytes or Edu-labelled $\Delta 113 p 53$-positive cardiomyocytes were rarely observed in the sham hearts (Fig. 2a). The results demonstrate that many $\triangle 113 p 53: G F P^{+}$cells near the 


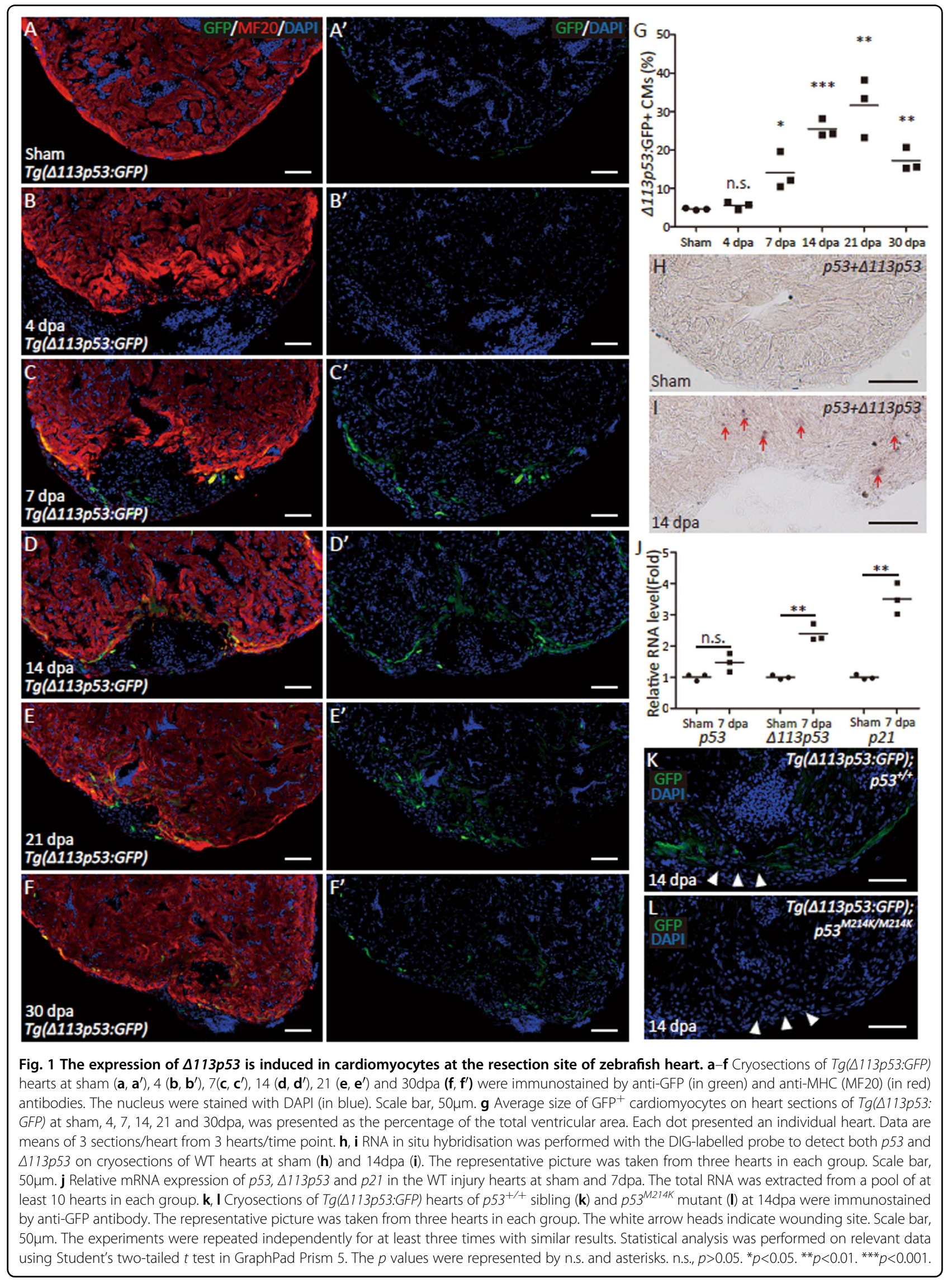




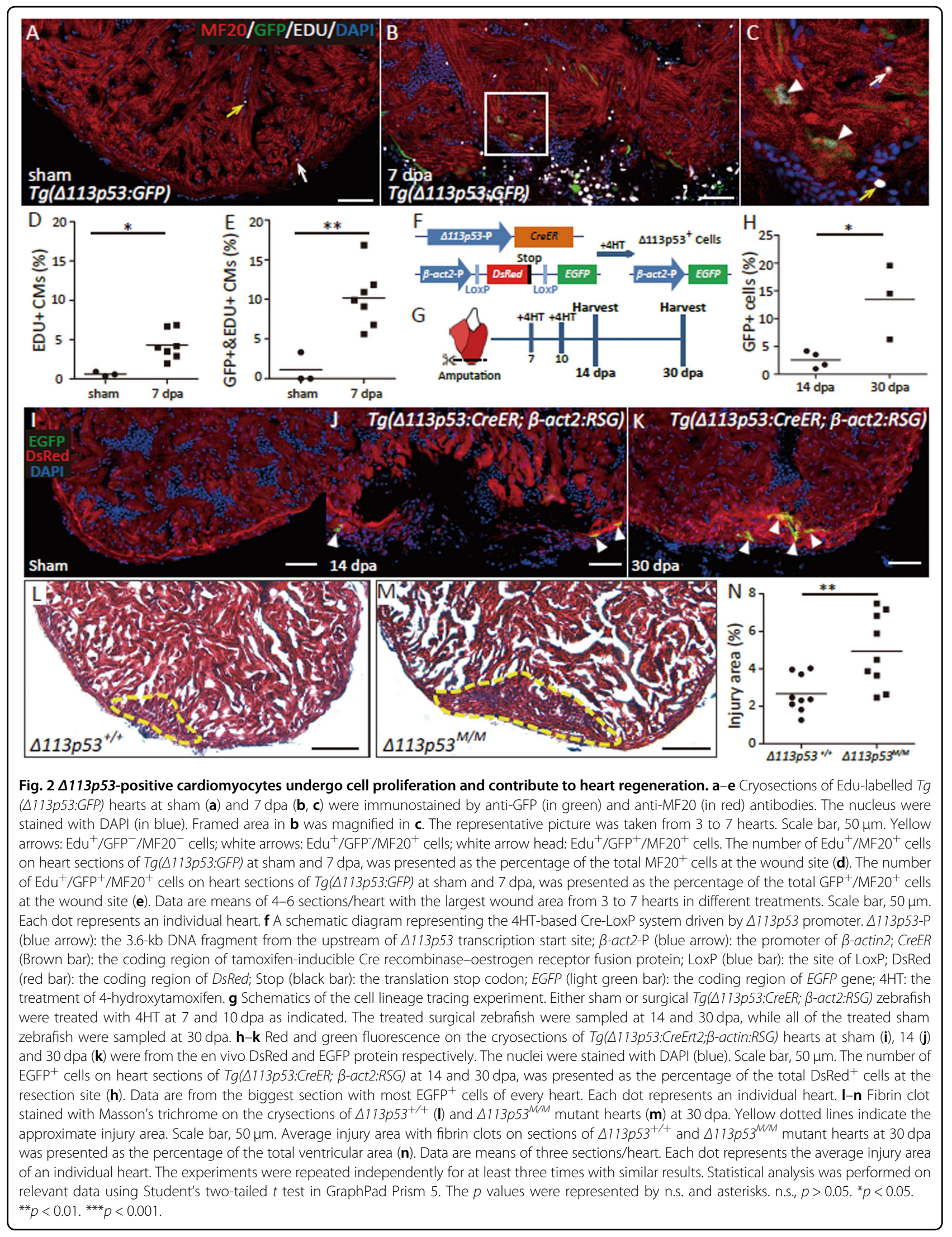


lateral edges of the wound have newly undergone DNA synthesis.

To investigate the dynamics of $\Delta 113 p 53$-positive cardiomyocytes in heart regeneration, a cell lineage tracing assay was performed. We generated $\operatorname{tg}(\triangle 113 p 53: C r e E R)$ transgenic zebrafish using a $3.6-\mathrm{kb}$ fragment of the $\triangle 113 p 53$ promoter to drive CreER (tamoxifen-inducible Cre recombinase-oestrogen receptor fusion protein) expression and crossed them with $\operatorname{tg}(\beta$-act2:RSG) zebrafish to generate $\operatorname{tg}(\triangle 113 p 53: C r e E R ; \beta$-act2:RSG) double transgenic fish (Fig. 2f). Our previous study revealed that the expression of $\Delta 113 \mathrm{p} 53$ is strongly induced upon treatment with DNA-damaging drugs ${ }^{40}$. To verify the utility of the double transgenic fish, the transgenic embryos were treated with either camptothecin (Campt, a DNA-damaging drug), 4-hydroxytamoxifen $(4 \mathrm{HT})$ or a combination of both. Western blot analysis showed that the expression of endogenous $\Delta 113 \mathrm{p} 53$ was induced by Campt but not by $4 \mathrm{HT}$ (Fig. S3a). Green fluorescence appeared in the transgenic embryos treated with the combination of Campt and 4HT (Fig. S3e) but not in the untreated embryos or the embryos treated with either drug alone (Fig. S3b-d). The results demonstrated that the double transgenic fish could be used to trace the induction of $\Delta 113 p 53$.

Next, we treated the sham zebrafish and adult double transgenic zebrafish subjected to surgery with $4 \mathrm{HT}$ at 7 and $10 \mathrm{dpa}$ (Fig. $2 \mathrm{~g}$ ), the time points preceding detectable of $\triangle 113 p 53$-driven GFP fluorescence in the injury site. At $14 \mathrm{dpa}$, a small number of $\mathrm{EGFP}^{+}$cardiomyocytes (2.6\%) were detected near the border of the wound in the $4 \mathrm{HT}$ treated $\operatorname{tg}(\triangle 113 p 53: C r e E R ; \beta$-act2:RSG) animals (Fig. 2j, h) but not in the sham controls (Fig. 2i). Moreover, the number of $\mathrm{EGFP}^{+}$cardiomyocytes significantly increased to $13.5 \%$ at $30 \mathrm{dpa}$ (Fig. $2 \mathrm{k}, \mathrm{h}$ ). These results indicate that $\Delta 113 p 53$-positive cardiomyocytes undergo cell proliferation and contribute to heart regeneration.

\section{Heart regeneration is impaired in $\Delta 113 p 53^{M / M}$ mutant zebrafish}

During zebrafish heart regeneration, a large clot of blood cells (most of them being erythrocytes) forms in the resection site after a few seconds of profuse bleeding from the ventricular lumen; these blood cells are replaced by fibrin beginning $2 \mathrm{dpa}$. Cardiomyocytes surround, penetrate and finally replace the fibrin clot from 9 to $30 \mathrm{dpa}^{2}$. The area of the injury containing the fibrin clot is a critical parameter for evaluating the quality of heart regeneration $^{49}$. To investigate the role of $\Delta 113 p 53$ in heart regeneration, we performed Masson's staining to compare the area of the injury containing the fibrin clot between the resected hearts of WT zebrafish and those of $\Delta 113 p 53^{M / M}$ mutant zebrafish. The $\Delta 113 p 53^{M / M}$ mutant generated in our previous study exhibits relatively normal development and carries an 11-bp deletion in a p53 responsive element in the $\Delta 113 p 53$ promoter located in the 4th intron of $p 53$, which abolishes the expression of $\triangle 113 p 53$ but does not influence the expression of fulllength $\mathrm{p} 53^{44}$. The results showed that there were no visible differences between uninjured $\Delta 113 p 53^{M / M}$ mutant and WT hearts (Fig. S4), which suggests that the expression of $\triangle 113 p 53$ in heart development is weak and that $\Delta 113 p 53$ plays a small role in heart development. However, the percentage of the injury area containing the fibrin clot was significantly larger in $\Delta 113 p 53^{M / M}$ mutant hearts (4.94\%) (Fig. 2m, n) than in WT hearts (2.67\%) (Fig. 2l, n) at $30 \mathrm{dpa}$. These results demonstrate that $\Delta 113 p 53$ is induced to promote heart regeneration.

\section{$\Delta 113 p 53$ has little effect on the activation of gata4-positive cardiomyocytes and their migration to the edge of the wound site}

The gata4-positive cardiomyocytes dedifferentiated from cardiomyocytes in the subepicardial ventricular layer migrate to the injury site and proliferate to contribute to zebrafish heart regeneration ${ }^{5,6}$. To investigate whether $\Delta 113 p 53$-positive cells were dedifferentiated cardiomyocytes, we generated $\operatorname{tg}(\Delta 113 p 53: m$ Cherry $)$ transgenic zebrafish by using a $3.6-\mathrm{kb}$ fragment of the $\Delta 113 p 53$ promoter to drive mCherry expression (Fig. S5) and crossed them with $\operatorname{Tg}$ (gata4:EGFP) zebrafish to obtain $t g$ ( $\triangle 113 p 53: m$ Cherry; gata4:EGFP) double transgenic fish. Immunostaining assays showed that mCherry was coexpressed with EGFP in some $\mathrm{EGFP}^{+}$cardiomyocytes near the wound site at $14 \mathrm{dpa}$ (Fig. 3a). These results demonstrate that $\Delta 113 p 53^{+}$cells are dedifferentiated cardiomyocytes.

Next, $\operatorname{Tg}$ (gata4:EGFP) transgenic reporter zebrafish were used to track newly regenerated cardiomyocytes in injured $\Delta 113 p 53^{M / M}$ mutant hearts. We found that there were no visible differences in the location or percentage of gata4positive cardiomyocytes in WT and $\Delta 113 p 53^{M / M}$ mutant hearts at $7 \mathrm{dpa}$ (Fig. 3b, c, f). Similar to those in the WT hearts, gata4-positive cardiomyocytes in $\Delta 113 p 53^{M / M}$ mutant hearts migrated to the edge of the wound site at $14 \mathrm{dpa}$ (Fig. 3d, e), although the percentage of gata4positive cardiomyocytes at the edge of the wound site was slightly lower in $\Delta 113 p 53^{M / M}$ mutant hearts than in WT hearts at $14 \mathrm{dpa}$ (Fig. 3d-f). However, unlike in WT hearts, gata4-positive cardiomyocytes were rarely observed in the intermediate zone of the wound area in $\Delta 113 p 53^{M / M}$ mutant hearts (Fig. 3d, e); it is unclear whether this phenomenon resulted from cardiomyocyte proliferation or from the penetration of gata4-positive cells. These results suggest that $\Delta 113 \mathrm{p} 53$ does not play a critical role in cardiomyocyte dedifferentiation or the migration of gata4positive cardiomyocytes from the outer compact layer of the ventricle to the edge of the wound site. 


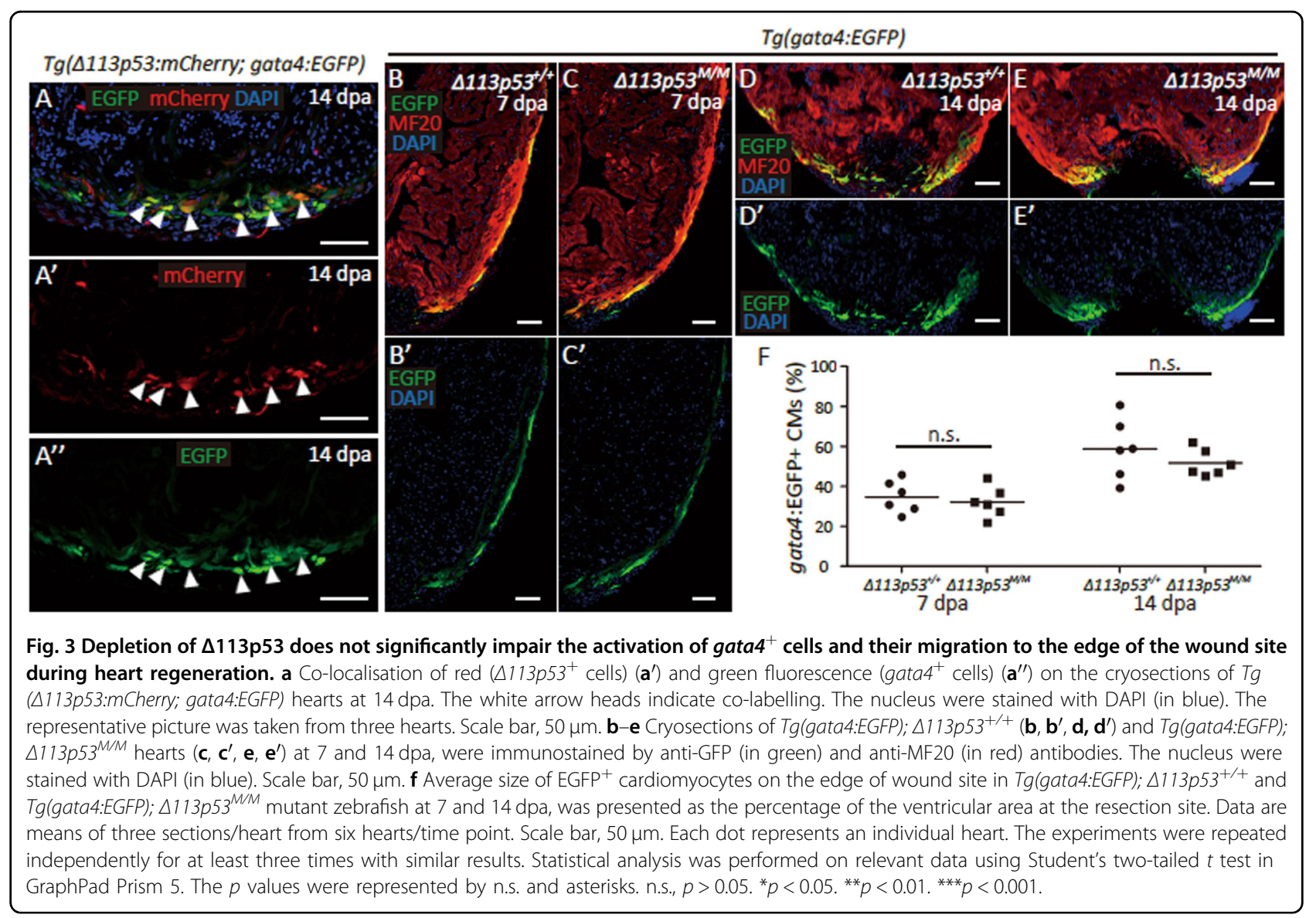

\section{$\Delta 113 p 53$ promotes heart regeneration by enhancing cardiomyocyte proliferation, but not by inhibiting cardiomyocyte apoptosis}

A recent study showed that cryoinjury triggers the DNA damage response during zebrafish heart regeneration ${ }^{49}$. Our previous studies revealed that $\Delta 133 \mathrm{p} 53$ is induced during cell reprogramming to promote reprogramming efficiency through its anti-apoptotic activity and ensure the genomic integrity of induced pluripotent stem cells by increasing DNA DSB repair ${ }^{50}$. To compare apoptotic activity and the DNA damage response between the ventricles of WT and $\Delta 113 p 53^{M / M}$ mutant hearts during regeneration, the $\operatorname{tg}(m y l 7: n D s R e d)$ transgenic line (in which the promoter of zebrafish myosin light chain 7 drives the expression of nuclear DsRed) was crossed onto the $\Delta 113 p 53^{M / M}$ mutant background. The TUNEL assay and immunostaining for $\gamma-\mathrm{H} 2 \mathrm{AX}$ (an early marker of the DNA damage response) were performed to analyse apoptotic cells and the DNA damage response, respectively. We found that there were only a few apoptotic cardiomyocytes and $\gamma$-H2AX-positive cardiomyocytes (co-stained with $\mathrm{nDsRed}$ ) in the wound site in both WT and $\Delta 113 p 53^{M / M}$ mutant hearts at $14 \mathrm{dpa}$ (Fig. S6). These results suggest that $15 \%$ resection of the ventricle does not trigger a strong DNA damage response in cardiomyocytes during heart regeneration.

To compare myocardial proliferation in the ventricles of WT and $\Delta 113 p 53^{M / M}$ mutant hearts during regeneration, we quantified injury-induced cardiomyocyte proliferation by counting $\mathrm{EdU}^{+} / \mathrm{Myl}^{+}$or $\mathrm{PCNA}^{+}$(the DNA replication marker proliferating cell nuclear antigen) $/ \mathrm{Myl}^{+}$ double-positive cardiomyocytes during heart regeneration. Compared to WT hearts, $\triangle 113 p 53^{M / M}$ mutant hearts harboured significantly fewer proliferating cardiomyocytes labelled with $\mathrm{EdU}^{+} / \mathrm{Myl}^{+}$at $14 \mathrm{dpa}$ (54\% of the number in WT hearts) (Fig. 4a, b, e) and with $\mathrm{PCNA}^{+} /$ $\mathrm{Myl}^{+}$at $7 \mathrm{dpa}$ (85\% of the number in WT hearts) (Fig. 4c, d, f). These data reveal that $\Delta 113 p 53$ is required for cardiomyocyte proliferation following injury.

$\Delta 113 p 53$ upregulates the expression of antioxidant genes to maintain redox homeostasis during heart regeneration

A recent study revealed that $\mathrm{H}_{2} \mathrm{O}_{2}$ is produced near the wound site of ventricles to promote heart regeneration ${ }^{16}$. Our previous study demonstrated that the human orthologue $\Delta 133 \mathrm{p} 53$ is induced in response to sub-toxic levels of ROS to promote cell proliferation by upregulating the expression of antioxidant genes ${ }^{46}$. Therefore, we 


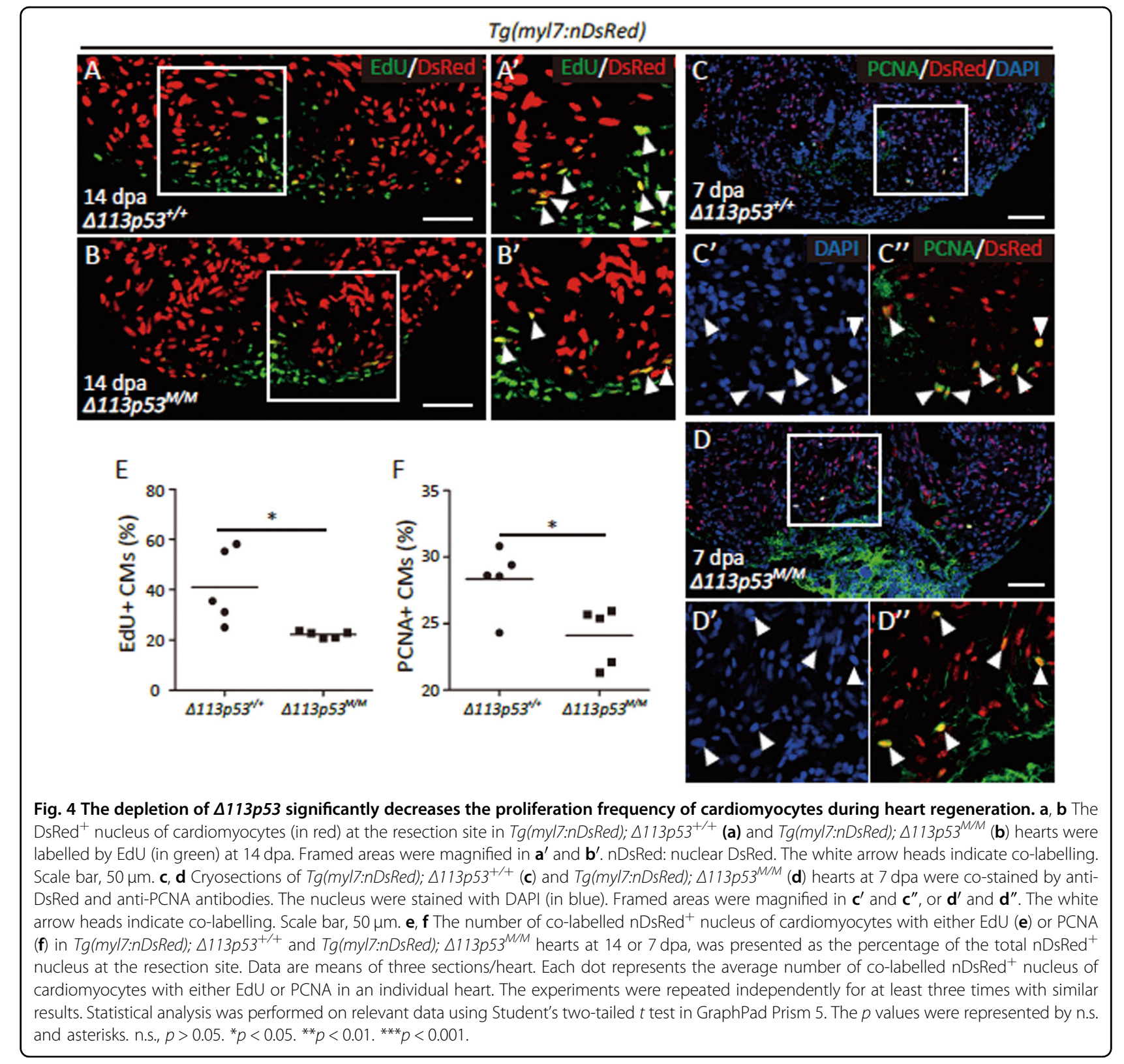

investigated whether the induction of $\Delta 113 p 53$ is related to maintaining redox homeostasis during heart regeneration. For this purpose, we treated $\operatorname{tg}(\triangle 113 p 53: G F P)$ zebrafish with diphenylene iodonium (DPI), an NADPH oxidase (Duox/Nox enzymes) inhibitor, to block the production of $\mathrm{H}_{2} \mathrm{O}_{2}{ }^{16}$ after amputation. The results showed that compared to control treatment, DPI treatment significantly reduced the percentage of $\Delta 113 p 53^{+}$ cardiomyocytes near the wound site at 7 and $14 \mathrm{dpa}$ (Fig. 5a-e), suggesting that the induction of $4113 p 53$ depends on elevation of ROS levels during heart regeneration.

Next, we determined the status of ROS in the injured hearts of both WT and $\Delta 113 p 53^{M / M}$ mutant zebrafish at different time points with the $\operatorname{tg}(m y l 7: H y P e r)$ transgene (in which the promoter of $m y l 7$ drives the expression of HyPer, a fluorescent protein-based $\mathrm{H}_{2} \mathrm{O}_{2}$ sensor) ${ }^{16}$. Similar to a previous study ${ }^{16}$, the level of $\mathrm{H}_{2} \mathrm{O}_{2}$ in the injured WT hearts started to increase at $3 \mathrm{dpa}$, decreased beginning at $14 \mathrm{dpa}$ and reached the basal level at $21 \mathrm{dpa}$ (Fig. 5f, h, j, l), whereas the $\mathrm{H}_{2} \mathrm{O}_{2}$ levels in the injured $\Delta 113 p 53^{M / M}$ mutant hearts were significantly higher than those in the injured WT hearts at 10.5 and 21 dpa (Fig. 5g, $\mathrm{i}, \mathrm{k}, \mathrm{l})$. These results suggest that depletion of $\Delta 113 p 53$ results in elevated levels of intracellular $\mathrm{H}_{2} \mathrm{O}_{2}$ during heart regeneration.

To investigate whether elevated ROS levels in the injured $\Delta 113 p 53^{M / M}$ mutant hearts were related to antioxidant genes, we examined the expression of six antioxidant genes 


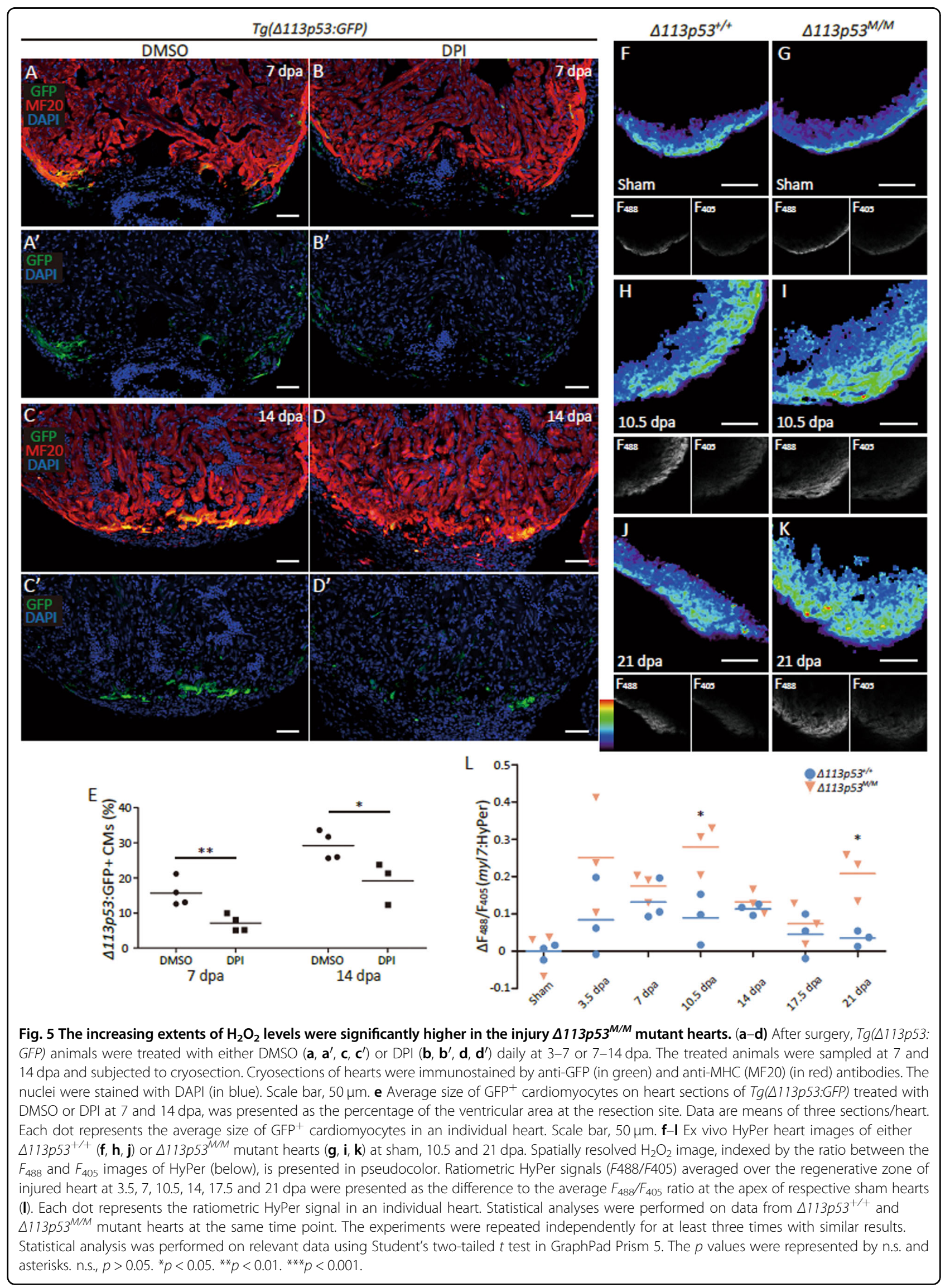


(p53 target genes), including gpx1a, sesn2, aldh4, sesn1, sod 1 and $\operatorname{sod} 2$, by using qRT-PCR. The expression of $g p x 1 a$ and sesn2, as well as the expression of $\Delta 113 p 53$ was significantly upregulated in the injured WT hearts (Fig. 6a-c) compared to the sham hearts at $14 \mathrm{dpa}$, whereas the expression of the remaining 4 genes was not significantly changed (Fig. S7). Interestingly, the expression of all six antioxidant genes in sham $\Delta 113 p 53^{M / M}$ mutant hearts was lower than that in sham WT hearts (Figs. 6b, c and S7). Furthermore, the induction of gpxla was not triggered in injured $\Delta 113 p 53^{M / M}$ mutant hearts at $14 \mathrm{dpa}$ (Fig. 6b), while the induction of $\operatorname{ses} n 2$ was significantly lower in injured $\Delta 113 p 53^{M / M}$ mutant hearts than in injured WT hearts at $14 \mathrm{dpa}$; however, the expression of sesn 2 was increased in injured $\Delta 113 p 53^{M / M}$ mutant hearts compared to sham $\Delta 113 p 53^{M / M}$ mutant hearts (Fig. 6c). These results
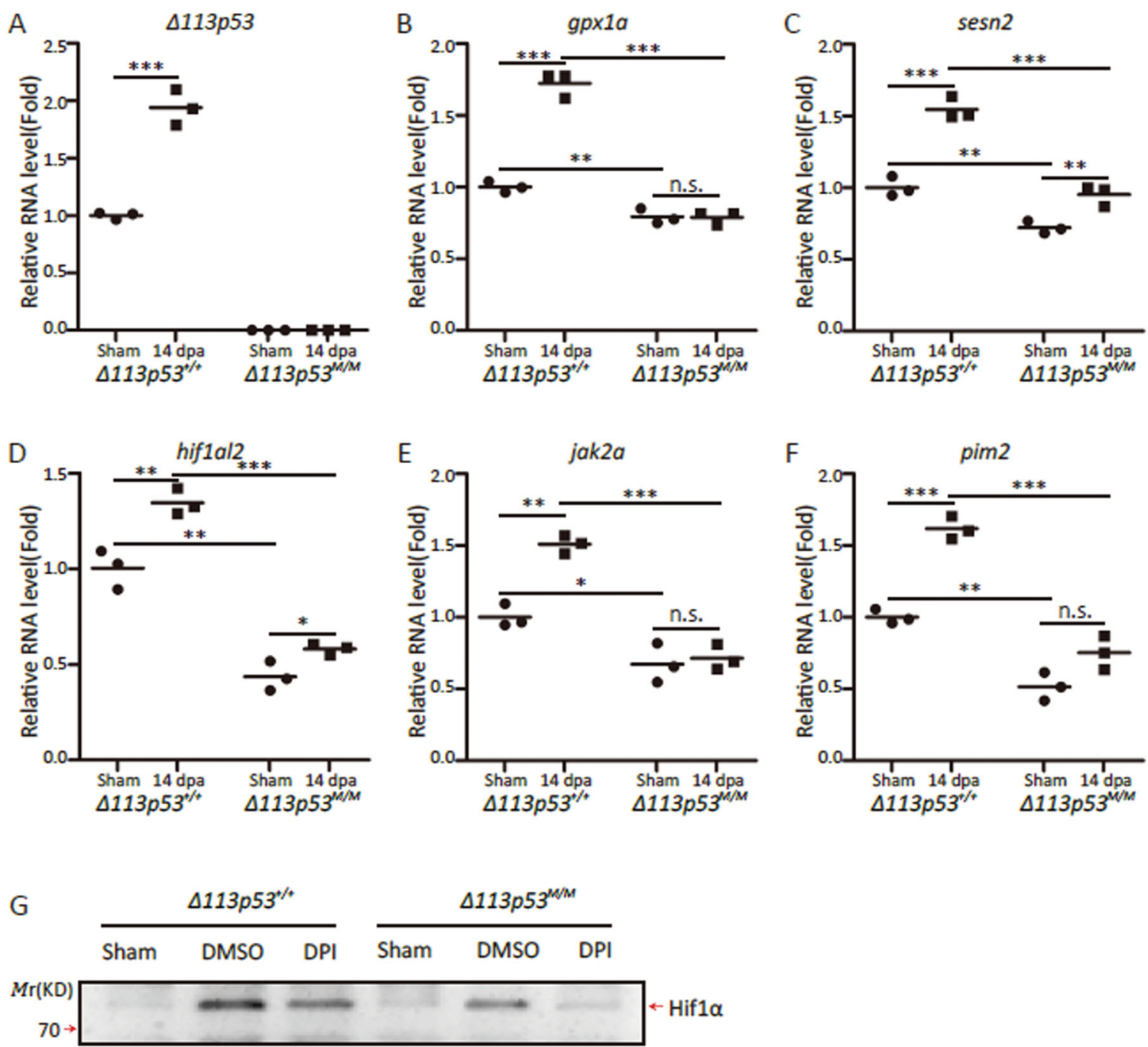

Gapdh

Fig. 6 Upregulation of antioxidant and proproliferative genes during heart regeneration is impaired in the injury $\Delta 113 p 53^{M / M}$ mutant hearts. a-f Relative mRNA expression of $\triangle 113 p 53(\mathbf{a})$, gpx $1 a(\mathbf{b})$, sesn $2(\mathbf{c})$, hif1 al2 (d), jak2a (e) and pim2 (f) in the $\Delta 113 p 53^{+/+}$and $\Delta 113 p 53^{M / M}$ hearts at sham and $14 \mathrm{dpa}$. The total RNA was extracted from a pool of at least 10 hearts in each group. $\mathbf{g}$ Western blot was performed to analyse the induction of zebrafish Hif1a in different samples as indicated. The $\Delta 113 p 53^{+/+}$and $\Delta 113 p 53^{M / M}$ mutant zebrafish with heart resection were treated with DPI from 3 to $7 \mathrm{dpa}$. Total protein was isolated from four hearts/treatment at $7 \mathrm{dpa}$ and subjected to western blot analysis. Gapdh was used as the protein loading control. The experiments were repeated independently for at least three times with similar results. Statistical analysis was performed on relevant data using Student's two-tailed $t$ test in GraphPad Prism 5. The $p$ values were represented by n.s. and asterisks. n.s., $p>0.05$. ${ }^{*} p<0.05 .{ }^{* *} p<0.01 .{ }^{* *} p<0.001$. 
demonstrate that the antioxidant response is triggered in wounded hearts and that $\Delta 113 p 53$ promotes the expression of antioxidant genes. This result also implies that the elevation of ROS levels in the injured $\Delta 113 p 53^{M / M}$ mutant hearts is due to lower expression of antioxidant genes.

Finally, we tried to gain insight into the role of elevated ROS levels in cardiomyocyte proliferation. ROS stress elicits the ATM-homodimer-Chk2 pathway to trigger the DNA damage response ${ }^{51}$. However, our results showed that apoptotic activity and the DNA damage response was rarely induced by $15 \%$ ventricular resection in both WT and $\Delta 113 p 53^{M / M}$ mutant hearts (Fig. S6). A previous study revealed that cardiac injury induces the hypoxia response in zebrafish ventricles, resulting in activation of Hif1 $\alpha$ signalling, which promotes cardiomyocyte proliferation by upregulating the expression of numerous proproliferative genes, including many components of the Jak-STAT pathway ${ }^{52}$. A number of studies have also documented that an increase in ROS levels can downregulate Hif1 $\alpha$ signalling ${ }^{53-55}$. Therefore, we evaluated the expression of three genes in the Hif1 $\alpha$ signalling pathway, including hif1al2 (hypoxia inducible factor 1 subunit alpha, like 2), jak2a and pim2 (two HIF1 $\alpha$ downstream genes), by qRT-PCR. Consistent with a previous study ${ }^{52}$, the expression of these three genes was upregulated in injured WT hearts (Fig. 6d-f) compared to sham hearts at $14 \mathrm{dpa}$. The expression of all three genes in sham $\Delta 113 p 53^{M / M}$ mutant hearts was lower than that in sham WT hearts (Fig. 6d-f). Although the expression of hiflal2 was also upregulated in injured $\Delta 113 p 53^{M / M}$ mutant hearts at 14dpa (Fig. 6d), the induction of two downstream genes, $j a k 2 a$ and $p i m 2$, was abolished in injured $\Delta 113 p 53^{M / M}$ mutant hearts at $14 \mathrm{dpa}$.(Fig. 6e, f). These results suggest that the elevation of ROS levels may repress cardiomyocyte proliferation through inactivating the Hif1 $\alpha$ signalling pathway.

To verify the activation of hifl $\alpha$ in heart regeneration and address if the hifl $\alpha$ activation is dependent on the ROS signal, we treated WT and $\Delta 113 p 53^{M / M}$ mutant zebrafish with DPI to block the production of $\mathrm{H}_{2} \mathrm{O}_{2}$ after amputation and analysed the level of Hif1 $\alpha$ protein at $7 \mathrm{dpa}$. The western blot analysis confirmed that the expression of Hif1 $\alpha$ was induced in both WT and $\Delta 113 p 53^{M / M}$ mutant resected hearts, compared to that in respective sham hearts (Fig. $6 \mathrm{~g}$ ). Interestingly, DPI treatment observably reduced the activation of Hifl $\alpha$ protein in both WT and $\Delta 113 p 53^{M / M}$ mutant resected hearts at $7 \mathrm{dpa}$ (Fig. $6 \mathrm{~g}$ ), suggesting that the induction of Hif1 $\alpha$ depends on elevation of ROS levels during heart regeneration.

\section{Discussion}

It is well documented that ROS are produced after tissue injury and play an important role in wound healing by initiating acute inflammation, clarifying infection and dead tissue, and mediating various intracellular signal transduction pathways ${ }^{5-58}$. However, when the level of ROS is beyond a cell's capacity to maintain redox homeostasis, oxidative stress occurs, which results in direct or indirect ROS-mediated damage to nucleic acids, proteins and lipids ${ }^{20,21}$. Therefore, ROS levels in cells are tightly controlled by antioxidant systems $^{22}$. P53 and its isoform $\Delta 133$ p53/ $\Delta 113$ p53 play a critical role in the maintenance of redox homeostasis by regulating the expression of antioxidant genes ${ }^{46}$. Interestingly, ROS are also generated during zebrafish heart regeneration to promote cardiomyocyte proliferation ${ }^{16}$, and the p53 signalling pathway is activated during cardiac regeneration in neonatal mice ${ }^{47}$. However, how redox homeostasis is maintained and whether p53 signalling plays a role in heart regeneration remain unclear.

In this report, we applied partial zebrafish ventricular resection to investigate the function of $4113 p 53$ in heart regeneration. Based on a p53-based genetic tracing system involving the insertion of a CreER cassette immediately after the first ATG of the full-length mouse p53 BAC clone (located in the second exon of p53), a previous study revealed that full-length $p 53$-positive cardiomyocytes are activated by injury in neonatal mice and undergo proliferation to contribute to heart regeneration ${ }^{47}$. In contrast, using $\Delta 113 p 53$ transgenic reporter fish, in situ hybridisation and qRT-PCR, we found that the transcription of $\Delta 113 p 53$, but not full-length $\mathrm{p} 53$, was induced in cardiomyocytes near the injury site in zebrafish ventricles (Fig. 1a-j). The induction of $\Delta 113 p 53$ was not observed in injured $p 53^{M 214 K}$ mutant hearts (Fig. 1k, l), which is consistent with $\triangle 113 p 53$ being a p53 target gene ${ }^{40}$. The discrepancy between the two studies in mouse and zebrafish may be explained by the fact that the mouse p 53 reporter system contains the first exon of $\mathrm{p} 53^{47}$, which might be the promoter for the mouse $\Delta 113 p 53 / \Delta 133 p 53$ orthologue. Next, we explored the function of $\Delta 113 p 53$ in heart regeneration in $\Delta 113 p 53^{M / M}$ mutants. Masson's staining showed that the area of the injury containing the fibrin clot was significantly increased in the wound site in $\triangle 113 p 53^{M /}$ ${ }^{M}$ mutant hearts (Fig. 2l-n) compared to WT hearts at $30 \mathrm{dpa}$, which demonstrates that heart regeneration is impaired in the $\Delta 113 p 53^{M / M}$ mutants. Although there were no observable differences in dedifferentiation to gata4-positive cardiomyocytes (Fig. 3b-f) or cardiomyocyte apoptosis between injured WT and $\Delta 113 p 53^{M / M}$ mutant hearts (Fig. S6c, d), the percentages of EDUlabelled cardiomyocytes and PCNA-labelled cardiomyocytes were significantly lower in injured $\triangle 113 p 53^{M / M}$ mutant hearts than in injured WT hearts (Fig. 4). These results reveal that $\Delta 113 p 53$ promotes heart regeneration by increasing cardiomyocyte proliferation. Further analysis showed that $\mathrm{H}_{2} \mathrm{O}_{2}$ levels in the injured $\Delta 113 p 53^{M / M}$ mutant hearts were significantly higher than those in the injured WT hearts (Fig. 5f-l) and that the increase in 
$\mathrm{H}_{2} \mathrm{O}_{2}$ levels was coincident with a decrease in antioxidant gene expression in the injured $\Delta 113 p 53^{M / M}$ mutant hearts (Fig. 6b, c). These results suggest that $\Delta 113 \mathrm{p} 53$ promotes cardiomyocyte proliferation by maintaining redox homeostasis.

Taken together, our findings demonstrate that although ROS signalling plays an important role in promoting heart regeneration ${ }^{16}$, the level of ROS should be tightly controlled. The induction of $\Delta 113 \mathrm{p} 53$ functions to maintain redox homeostasis by promoting antioxidant gene expression.

Oxidative stress has been implicated in human cardiac diseases, including ischaemia-reperfusion (IR), myocardial infarction (MI) and heart failure ${ }^{57,59}$. ROS are produced in two stages, namely, ischaemia and reperfusion, at low and high levels, respectively ${ }^{60}$. ROS play a dual role in tissue injuries, as massive amounts of mitochondrial ROS induce apoptosis and necrosis of cells ${ }^{61}$, whereas moderate levels of ROS promote cell survival and proliferation ${ }^{16,62,63}$. Therefore, maintaining redox homeostasis plays an important role in the mechanisms of and therapeutic strategies for cardiac diseases. It has also been reported that during pressure overload, the activation of full-length p53 has a crucial function in the transition from cardiac hypertrophy to heart failure by repressing Hif1 activity ${ }^{64}$. Here, we demonstrate that $\Delta 113 \mathrm{p} 53$ is induced by ROS during zebrafish heart regeneration and functions to promote cardiomyocyte proliferation by maintaining redox homeostasis and Hifl $\alpha$ activity. Our results suggest that the expression of $\Delta 133 p 53$ may also be activated during IR and protect patients from IR-induced heart failure.

\section{Methods and materials Zebrafish lines}

Zebrafish were raised and maintained in standard zebrafish units at Zhejiang University as described previously ${ }^{44}$. The $\operatorname{Tg}(\triangle 113 p 53: G F P)$ transgenic line and $\Delta 113 p 53^{M / M}$ mutant zebrafish were generated in our previous studies ${ }^{40,44}$. A $3.6-\mathrm{kb}$ fragment of the $\Delta 113 p 53$ promoter $^{40}$ was used to create the $\operatorname{Tg}(\triangle 113 p 53: C r e E R)$ and $\operatorname{tg}(\triangle 113 p 53: m C h e r r y)$ transgenic lines on the $\mathrm{AB}$ genetic background through Tol2-based transgenesis ${ }^{40}$. $p 53^{M 214 K}$ mutant $^{48}, \operatorname{Tg}(\beta \text {-act 2:RSG })^{5}, \operatorname{Tg}(\text { gata4:EGFP })^{65}$, $T g(m y l 7: n D s R e d)^{66}$ and $\operatorname{Tg}(m y l 7: H y P e r)^{16}$ zebrafish were generated by different labs as previously reported.

\section{Ethics statement}

All animal procedures were performed in full accordance with the requirements of the Regulation for the Use of Experimental Animals of Zhejiang Province. This work was specifically approved by the Animal Ethics Committee of the School of Medicine, Zhejiang University (ethics code permit no. ZJU20190012).

\section{Adult zebrafish heart resection}

Ventricular surgery was performed on 5- to 10-monthold zebrafish according to previously described procedures $^{2}$. Briefly, zebrafish were anaesthetised with $0.02 \%$ Tricaine and then subjected to $\sim 15 \%$ ventricular amputation at the apex with scissors.

\section{Quantitative real-time reverse transcriptional PCR}

Hearts were freshly isolated from anaesthetised zebrafish subjected to sham surgery or resection at different time points. The outflow tracts and atriums were removed from the isolated hearts. Total RNA was extracted from $\sim 10$ isolated ventricles from each group using a homogeniser (JXFSTPRP-24, Shanghai Jingxin) in Invitrogen TRIzol reagent (Cat No. 15596026). Isolated RNA was treated with DNaseI (NEB, M0303S) prior to reverse transcription and purified through lithium chloride. First-strand cDNA was synthesised using M-MLV Reverse Transcriptase (Invitrogen, C28025021). The reaction was performed using a CFX96 $^{\mathrm{TM}}$ Real-Time System (Bio-Rad) with AceQ qPCR SYBR Green (Vazyme, Q111-02) according to the manufacturer's instructions. Total RNA levels were normalised to the level of $\beta$-actin. Statistics were obtained from three repeats. The primer sequences of the analysed genes are listed in Table S1.

\section{In situ hybridisation}

For the in situ hybridisation assay, isolated zebrafish hearts were fixed in 4\% PFA for 2 days before cryosectioned. The probes were generated by NEB T7 RNA Polymerase (M0251S) and Roche DIG RNA Labelling Mix (11277073910) from a $\Delta 113 p 53-p C S 2^{+}$plasmid constructed in our previous study ${ }^{44}$. Staining was performed with Anti-Digoxigenin-AP (Roche, 11093274910) and the BCIP/NBT Alkaline Phosphatase Colour Development Kit (Beyotime Biotechnology, C3206).

\section{Ex vivo intact heart imaging}

Ex vivo $\mathrm{Tg}(\mathrm{myl7:HyPer})$ heart imaging and image processing were performed according to previously described procedures ${ }^{16}$. Briefly, images were taken under an Olympus FV1000 upright confocal microscope, and the HyPer 488/405 ratio was calculated based on the integrated optic density using Adobe Photoshop CS5.

\section{EdU incorporation assay and small-molecule treatment}

For the EdU incorporation assay, $15 \mu \mathrm{L}$ of $100 \mathrm{mM}$ EdU (Invitrogen, A10044) was injected once daily into the abdominal cavity of each animal that underwent surgery for 3 or 7 days until the hearts were collected at 7 or $14 \mathrm{dpa}$. The hearts were then fixed for cryosectioning. EdU staining was performed using Azide Alexa Fluor 647 (Invitrogen, A10277). 
For DPI treatment, $50 \mu \mathrm{L}$ of $10 \mu \mathrm{M}$ DPI (Sigma, D2926) was injected daily into the thoracic cavity of each animal that underwent surgery beginning 3 or $7 \mathrm{dpa}$ until the hearts were collected at 7 or $14 \mathrm{dpa}^{16}$.

For the cell lineage tracing assay, $\operatorname{tg}(\triangle 113 p 53$ :CreER; $\beta$-act2:RSG) fish subjected to sham surgery or surgery were bathed in $3 \mu \mathrm{M} 4 \mathrm{HT}$ (Sigma, H7904) for $24 \mathrm{~h}$ at 7 and $10 \mathrm{dpa}$ as previously described ${ }^{15}$.

\section{Western blot, immunostaining and histological methods}

For the western blot assay, a zebrafish p53 monoclonal antibody was generated by HuaAn Biotechnology (Hangzhou, China) as previously described ${ }^{67}$. A human HIF1 $\alpha$ antibody (BOSTER, A00013-1) was used to detect zebrafish Hifla. A $\beta$-actin antibody (Huabio, R1207-1) was used as the protein loading control for the experiments in embryonic stages. A Gapdh antibody (HuaBio, R1208-3) was used as the protein loading control for the experiments in zebrafish heart regeneration. The secondary antibodies were HRP-conjugated goat anti-mouse IgG (Huabio, HA1006) and HRP-conjugated goat antirabbit IgG (Huabio, HA1001).

Zebrafish hearts were fixed, cryosectioned $(14 \mu \mathrm{m})$ as described previously ${ }^{2}$ and then subjected to immunostaining. The primary antibodies were anti-GFP (Abcam, ab13970), anti-MYH1E (MF20; Developmental Studies Hybridoma Bank, AB 2147781), anti-PCNA (Sigma, P8825), anti-DsRed (Clontech, 632496) and anti-H2A. XS139ph (Genetex, GTX127340). The secondary antibodies were Alexa Fluor 488-conjugated anti-chicken IgY H\&L (Abcam, ab150169), Alexa Fluor 647-conjugated anti-mouse IgG H\&L (Abcam, ab150115), Dylight 549conjugated anti-rabbit IgG H\&L (EarthOx, E032320) and Alexa Fluor 647-conjugated anti-rabbit IgG H\&L (Abcam, ab150143). Nuclei were stained with DAPI (BYT, C1002).

Masson's staining was performed on cryosections using trichrome Masson's staining solution from Servicebio (G1006). Myosin was visualised as red, and fibrin was visualised as blue. Images were captured under an Olympus BX53 microscope with a camera from Qimaging MicroPublisher 5.0 RTV.

\section{TUNEL assay}

The TUNEL assay was performed on freshly prepared cryosections of $\operatorname{tg}(\mathrm{myl7}: n D s R e d)$ zebrafish hearts using a fluorescein-based Roche In Situ Cell Death Detection Kit (Cat No. 11684795910).

\section{Quantification and statistical analysis}

Sample sizes were designed based on routine genetic analysis in zebrafish studies. The investigators were blinded to group allocation during data collection and analysis. No data were excluded from the analyses. All samples were randomly selected.

\section{Acknowledgements}

We thank Dr. Peidong Han for his kind help with processing images of ex vivo HyPer hearts. We also thank Drs. Chenglu Xiao, Nannan Chang, Meijun Pang, Mengmeng Huang and Yu Zhang for their generous gifts of protocols and/or transgenic zebrafish lines. This work was supported by the National Key R\&D Program of China (2018YFA0801000, 2018YFA0800500 and 2017YFA0504501), the National Natural Science Foundation of China (31871500) and the Fundamental Research Funds for the Central Universities.

\section{Author details}

${ }^{1}$ MOE Key Laboratory of Biosystems Homeostasis \& Protection and Innovation Center for Cell Signaling Network, College of Life Sciences, Zhejiang University, 310058 Hangzhou, China. ${ }^{2}$ College of Animal Sciences, Zhejiang University, 310058 Hangzhou, China. Institute of Molecular Medicine, Beijing Key Laboratory of Cardiometabolic Molecular Medicine, and State Key Laboratory of Natural and Biomimetic Drugs, Peking University, 100871 Beijing, China.

${ }^{4}$ Department of Plastic Surgery, Sir Run Run Shaw Hospital, Zhejiang University School of Medicine, No. 3 Qingchun Road East, 310016 Hangzhou, China

Conflict of interest

The authors declare that they have no conflict of interest.

\section{Publisher's note}

Springer Nature remains neutral with regard to jurisdictional claims in published maps and institutional affiliations.

Supplementary Information accompanies this paper at (https://doi.org/ 10.1038/s41419-020-02781-7).

Received: 27 November 2019 Revised: 8 July 2020 Accepted: 13 July 2020 Published online: 23 July 2020

\section{References}

1. Lallukka, T., Millear, A., Pain, A., Cortinovis, M. \& Giussani, G. GBD 2015 Mortality and Causes of Death Collaborators. Global, regional, and national life expectancy, all-cause mortality, and cause-specifi c mortality for 249 causes of death, 1980-2015: a systematic analysis for the Global Burden of Disease Study 2015 (vol 388, pg 1459, 2016). Lancet 389, E1-E1 (2017).

2. Poss, K. D., Wilson, L. G. \& Keating, M. T. Heart regeneration in zebrafish. Science 298, 2188-2190 (2002).

3. Porrello, E. R. et al. Transient regenerative potential of the neonatal mouse heart. Science 331, 1078-1080 (2011).

4. Gonzalez-Rosa, J. M., Martin, V., Peralta, M., Torres, M. \& Mercader, N. Extensive scar formation and regression during heart regeneration after cryoinjury in zebrafish. Development 138, 1663-1674 (2011).

5. Kikuchi, K. et al. Primary contribution to zebrafish heart regeneration by gata4 (+) cardiomyocytes. Nature 464, 601-605 (2010).

6. Gupta, V. et al. An injury-responsive gata4 program shapes the zebrafish cardiac ventricle. Curr. Biol. 23, 1221-1227 (2013).

7. Raya, A. et al. Activation of Notch signaling pathway precedes heart regeneration in zebrafish. Proc. Natl Acad. Sci. USA 100, 11889-11895 (2003).

8. Zhao, L. et al. Notch signaling regulates cardiomyocyte proliferation during zebrafish heart regeneration. Proc. Natl Acad. Sci. USA 111, 1403-1408 (2014).

9. $W u, C$. C. et al. Spatially resolved genome-wide transcriptional profiling identifies BMP signaling as essential regulator of zebrafish cardiomyocyte regeneration. Dev. Cell 36, 36-49 (2016).

10. Lien, C. L., Schebesta, M., Makino, S., Weber, G. J. \& Keating, M. T. Gene expression analysis of zebrafish heart regeneration. PLoS Biol. 4, e260 (2006).

11. Kim, J. et al. PDGF signaling is required for epicardial function and blood vessel formation in regenerating zebrafish hearts. Proc. Natl Acad. Sci. USA 107, 17206-17210 (2010).

12. Lepilina, A. et al. A dynamic epicardial injury response supports progenitor cell activity during zebrafish heart regeneration. Cell 127, 607-619 (2006).

13. Kikuchi, $K$. et al. Retinoic acid production by endocardium and epicardium is an injury response essential for zebrafish heart regeneration. Dev. Cell 20, 397-404 (2011). 
14. Gemberling, M., Karra, R., Dickson, A. L. \& Poss, K. D. Nrg1 is an injury-induced cardiomyocyte mitogen for the endogenous heart regeneration program in zebrafish. Elife 4, e05871 (2015).

15. Xiao, C. et al. Chromatin-remodelling factor Brg1 regulates myocardial proliferation and regeneration in zebrafish. Nat. Commun. 7, 13787 (2016).

16. Han, P. et al. Hydrogen peroxide primes heart regeneration with a derepression mechanism. Cell Res. 24, 1091-1107 (2014).

17. Terada, L. S. Specificity in reactive oxidant signaling: think globally, act locally. J. Cell Biol. 174, 615-623 (2006)

18. Takahashi, A. et al. Mitogenic signalling and the p16INK4a-Rb pathway cooperate to enforce irreversible cellular senescence. Nat. Cell Biol. 8 1291-1297 (2006).

19. Weinberg, F. \& Chandel, N. S. Reactive oxygen species-dependent signaling regulates cancer. Cell. Mol. Life Sci. 66, 3663-3673 (2009).

20. Valko, M. et al. Free radicals and antioxidants in normal physiological functions and human disease. Int. J. Biochem. Cell Biol. 39, 44-84 (2007).

21. Cooke, M. S., Evans, M. D., Dizdaroglu, M. \& Lunec, J. Oxidative DNA damage: mechanisms, mutation, and disease. FASEB J. 17, 1195-1214 (2003).

22. Ladelfa, M. F., Toledo, M. F., Laiseca, J. E. \& Monte, M. Interaction of p53 with tumor suppressive and oncogenic signaling pathways to control cellular reactive oxygen species production. Antioxid. Redox Signal. 15, 1749-1761 (2011).

23. Hafsi, H. \& Hainaut, P. Redox control and interplay between p53 isoforms: roles in the regulation of basal p53 levels, cell fate, and senescence. Antioxid. Redox Signal. 15, 1655-1667 (2011).

24. Vigneron, A. \& Vousden, K. H. p53, ROS and senescence in the control of aging. Aging 2, 471-474 (2010).

25. Holley, A. K. Dhar, S. K. \& St Clair, D. K. Manganese superoxide dismutase vs p53 Regulation of mitochondrial ROS. Mitochondrion 10, 649-661 (2010).

26. Liu, B., Chen, Y. M. \& Clair, D. K. S. ROS and p53: a versatile partnership. Free Radic. Biol. Med. 44, 1529-1535 (2008).

27. Tomko, R. J., Bansal, P. \& Lazo, J. S. Airing out an antioxidant role for the tumor suppressor p53. Mol. Inter. 6, 23-25 (2006).

28. Stambolsky, P. et al. Regulation of AIF expression by p53. Cell Death Differ. 13 2140-2149 (2006).

29. Matoba, S. et al. p53 regulates mitochondrial respiration. Science $\mathbf{3 1 2}$, 1650-1653 (2006).

30. Sablina, A. A. et al. The antioxidant function of the p53 tumor suppressor. Nat. Med. 11, 1306-1313 (2005)

31. Brand, K. A. \& Hermfisse, U. Aerobic glycolysis by proliferating cells: a protective strategy against reactive oxygen species. FASEB J. 11, 388-395 (1997).

32. Chance, B., Sies, H. \& Boveris, A. Hydroperoxide metabolism in mammalian organs. Physiol. Rev. 59, 527-605 (1979).

33. Pinton, $\mathrm{P}$. et al. Protein kinase $\mathrm{C}$ beta and prolyl isomerase 1 regulate mitochondrial effects of the life-span determinant p66Shc. Science 315, 659-663 (2007).

34. Liochev, S. I. \& Fridovich, I. The effects of superoxide dismutase on $\mathrm{H}_{2} \mathrm{O} 2$ formation. Free Radic. Biol. Med. 42, 1465-1469 (2007).

35. Dhar, S. K., Xu, Y., Chen, Y. \& St Clair, D. K. Specificity protein 1-dependent p53mediated suppression of human manganese superoxide dismutase gene expression. J. Biol. Chem. 281, 21698-21709 (2006).

36. Chen, J. et al. Loss of function of def selectively up-regulates Delta113p53 expression to arrest expansion growth of digestive organs in zebrafish. Genes Dev. 19, 2900-2911 (2005).

37. Bourdon, J. C. et al. p53 isoforms can regulate p53 transcriptional activity. Gene Dev. 19, 2122-2137 (2005)

38. Aoubala, M. et al. p53 directly transactivates Delta133p53alpha, regulating cell fate outcome in response to DNA damage. Cell Death Differ. 18, 248-258 (2011).

39. Marcel, V. et al. p53 regulates the transcription of its Delta 133 p53 isoform through specific response elements contained within the TP53 P2 internal promoter. Oncogene 29, 2691-2700 (2010).

40. Chen, J. et al. p53 isoform delta113p53 is a p53 target gene that antagonizes p53 apoptotic activity via BclxL activation in zebrafish. Genes Dev. 23, 278-290 (2009).

41. Ou, Z., Yin, L., Chang, C. Q., Peng, J. R. \& Chen, J. Protein interaction between p53 and delta 113p53 is required for the anti-apoptotic function of delta 113p53. J. Genet. Genomics 41, 53-62 (2014).
42. Fujita, K. et al. p53 isoforms Delta133p53 and p53beta are endogenous regulators of replicative cellular senescence. Nat. Cell Biol. 11, 1135-1142 (2009).

43. Mondal, A. M. et al. p53 isoforms regulate aging- and tumor-associated replicative senescence in T lymphocytes. J. Clin. Invest. 123, 5247-5257 (2013).

44. Gong, L. et al. p53 isoform Delta113p53/Delta133p53 promotes DNA doublestrand break repair to protect cell from death and senescence in response to DNA damage. Cell Res. 25, 351-369 (2015).

45. Gong, H. J. et al. p73 coordinates with Delta 133p53 to promote DNA doublestrand break repair. Cell Death Differ. 25, 1063-1079 (2018).

46. Gong, L., Pan, X., Yuan, Z. M., Peng, J. \& Chen, J. p53 coordinates with Delta133p53 isoform to promote cell survival under low-level oxidative stress. J. Mol. Cell Biol. 8, 88-90 (2016).

47. Xiao, Q. et al. A p53-based genetic tracing system to follow postnatal cardiomyocyte expansion in heart regeneration. Development $\mathbf{1 4 4}$ 580-589 (2017).

48. Berghmans, S. et al. tp53 mutant zebrafish develop malignant peripheral nerve sheath tumors. Proc. Natl Acad. Sci. USA 102, 407-412 (2005).

49. Bednarek, D. et al. Telomerase is essential for zebrafish heart regeneration. Cell Rep. 12, 1691-1703 (2015).

50. Gong, L. et al. p53 isoform Delta 133 p53 promotes efficiency of induced pluripotent stem cells and ensures genomic integrity during reprogramming. Sci. Rep. 6, 37281 (2016)

51. Ray, P. D., Huang, B. W. \& Tsuji, Y. Reactive oxygen species (ROS) homeostasis and redox regulation in cellular signaling. Cell Signal. 24, 981-990 (2012).

52. Jopling, C., Sune, G., Faucherre, A., Fabregat, C. \& Belmonte, J. C. I. Hypoxia induces myocardial regeneration in zebrafish. Circulation 126, 3017-U3435 (2012).

53. Callapina, M., Zhou, J., Schmid, T., Kohl, R. \& Brune, B. NO restores HIF-1 alpha hydroxylation during hypoxia: role of reactive oxygen species. Free Radic. Biol. Med. 39, 925-936 (2005).

54. Kohl, R., Zhou, J. \& Brune, B. Reactive oxygen species attenuate nitric-oxidemediated hypoxia-inducible factor-1 alpha stabilization. Free Radic. Biol. Med. 40, 1430-1442 (2006)

55. Chang, T. C. et al. Stabilization of hypoxia-inducible factor-1 alpha by prostacyclin under prolonged hypoxia via reducing reactive oxygen species level in endothelial cells. J. Biol. Chem. 280, 36567-36574 (2005).

56. Niethammer, P., Grabher, C., Look, A. T. \& Mitchison, T. J. A tissue-scale gradient of hydrogen peroxide mediates rapid wound detection in zebrafish. Nature 459, 996-999 (2009).

57. Bagheri, F. et al. Reactive oxygen species-mediated cardiac-reperfusion injury: Mechanisms and therapies. Life Sci. 165, 43-55 (2016).

58. Dunnill, $C$. et al. Reactive oxygen species (ROS) and wound healing: the functional role of ROS and emerging ROS-modulating technologies for augmentation of the healing process. Int. Wound J. 14, 89-96 (2017).

59. Braunersreuther, V. \& Jaquet, V. Reactive oxygen species in myocardial reperfusion injury: from physiopathology to therapeutic approaches. Curr. Pharm. Biotechnol. 13, 97-114 (2012).

60. Kevin, L. G., Novalija, E. \& Stowe, D. F. Reactive oxygen species as mediators of cardiac injury and protection: the relevance to anesthesia practice. Anesth. Analg. 101, 1275-1287 (2005).

61. Murphy, E. \& Steenbergen, C. Mechanisms underlying acute protection from cardiac ischemia-reperfusion injury. Physiol. Rev. 88, 581-609 (2008).

62. Alizadeh, A. M. Faghihi, M., Sadeghipour, H. R., Mohammadghasemi, F. \& Khori, $\checkmark$. Role of endogenous oxytocin in cardiac ischemic preconditioning. Regul. Pept. 167, 86-90 (2011).

63. Bernardi, P. \& Di Lisa, F. The mitochondrial permeability transition pore: Molecular nature and role as a target in cardioprotection. J. Mol. Cell. Cardiol. 78, 100-106 (2015)

64. Sano, M. et al. p53-induced inhibition of Hif-1 causes cardiac dysfunction during pressure overload. Nature 446, 444-448 (2007).

65. Heicklen-Klein, A. \& Evans, T. T-box binding sites are required for activity of a cardiac GATA-4 enhancer. Dev. Biol. 267, 490-504 (2004).

66. Mably, J. D., Mohideen, M. A., Burns, C. G., Chen, J. N. \& Fishman, M. C. heart of glass regulates the concentric growth of the heart in zebrafish. Curr. Biol. 13 2138-2147 (2003)

67. Tao, T. et al. Def defines a conserved nucleolar pathway that leads p53 to proteasome-independent degradation. Cell Res. 23, 620-634 (2013). 